NSF - ITP $-01-158$

\title{
ARITHMETIC OF CALABI-YAU VARIETIES AND RATIONAL CONFORMAL FIELD THEORY
}

\author{
RolF SCHIMmRIGK \\ Georgia Southwestern State University \\ 800 Wheatley St, Americus, GA 31709
}

\begin{abstract}
:
It is proposed that certain techniques from arithmetic algebraic geometry provide a framework which is useful to formulate a direct and intrinsic link between the geometry of Calabi-Yau manifolds and the underlying conformal field theory. Specifically it is pointed out how the algebraic number field determined by the fusion rules of the conformal field theory can be derived from the number theoretic structure of the cohomological Hasse-Weil L-function determined by Artin's congruent zeta function of the algebraic variety. In this context a natural number theoretic characterization arises for the quantum dimensions in this geometrically determined algebraic number field.
\end{abstract}

•email: netahu@yahoo.com,rks@canes.gsw.edu 


\section{Introduction}

In a stunning development Gepner suggested in 1987 that Calabi-Yau varieties are closely related to particular types of two-dimensional conformal field theories with integral central charges [1]. For the simplest class of models defined by weighted projective Brieskorn-Pham threefold varieties this relation might be formulated in a preliminary way as follows

$$
\mathbb{P}_{\left(k_{1}, k_{2}, k_{4}, k_{4}, k_{5}\right)}[d] \ni X=\left\{p\left(z_{i}\right)=\sum_{i} z_{i}^{d / k_{i}}=0\right\} \sim \bigotimes_{i=1}^{5} \mathrm{SU}(2)_{\frac{d}{k_{i}}-2} \text {. }
$$

Here the levels $\ell=\left(\frac{d}{k_{i}}-2\right)$ of the $\mathrm{SU}(2)_{\ell}$ conformal theories on the rhs are determined by the weights $k_{i}$ of the weighted projective space, denoted by $\mathbb{P}_{\left(k_{1}, \ldots, k_{5}\right)}$, and the degree $d:=\sum_{i} k_{i}$ of the defining polynomial of the variety $X$. For Brieskorn-Pham varieties the quotients $d / k_{i}$ are natural numbers. On the conformal field theory side we implicitly assume the GSO projection and that the affine characters of the partition function of the individual conformal factors are chosen to be the diagonal ones. The complete class of these models has been determined in [2, 3].

Gepner provided support for this relation by computing the massless spectrum of his theories and by showing that in two instances they agreed with the cohomology groups of known Calabi-Yau threefolds [4, 5]. Crucial insight into this surprising relation was obtained by Martinec [6] and Lerche, Vafa, and Warner [7, 8] who noted that an intermediate framework is provided by the concept of a Landau-Ginzburg theory. The latter defines a mean-field theory of the exactly solvable tensor model. In particular the important paper [9] by Vafa illuminated both the computation of the conformal field theory spectrum as well as providing effective insight into the cohomology of algebraic variety. Vafa's insight in particular made it possible to systematically investigate the relation between conformal field theory spectra and the cohomology of algebraic varieties and to extend this comparison to the complete class of models including non-diagonal affine invariants of the partition functions [2, 3]. Later more detailed insight into the nature of this relation via the intermediary Landau-Ginzburg theory was obtained by Witten 10 . 
At present, however, neither a direct nor a mathematically rigorous framework exists which allows to establish relations between algebraic varieties and conformal field theories. Furthermore the Landau-Ginzburg framework, important as it has been, is incomplete and does not allow to derive key ingredients of the conformal field theory. One might instead hope for a framework in which it is possible to derive the essential ingredients of the conformal field theory directly from the algebraic variety itself, without the intermediate Landau-Ginzburg formulation. A priori it might appear unlikely that such an approach exists because numbers associated to algebraic varieties are usually integers (such as dimension, cohomology dimensions and indices associated to complexes), whereas the numbers appearing in the underlying exactly solvable conformal field theory are usually rational numbers (such as the central charge and the anomalous dimensions).

It is the purpose of this article to suggest that an intrinsic, direct, and mathematically rigorous framework which allows to derive certain conformal field theoretic quantities directly from the algebraic Calabi-Yau variety is provided by arithmetic algebraic geometry. In this framework algebraic varieties $X$ are defined not over a continuous field, like the real numbers $\mathbb{R}$, or the complex number field $\mathbb{C}$, but over discrete finite fields, denoted by $\mathbb{F}_{q}$, where $q \in \mathbb{N}$ denotes the number of elements of the field. The particular field of reduction of the variety is specified by writing $X / \mathbb{F}_{q}$, leading to a reduced variety consisting of a finite number of points.

The choice of any finite $q$ would appear to be arbitrary, and physically ill-motivated. For small $q$ the field $\mathbb{F}_{q}$ would define a large scale lattice structure which one might expect to provide only rough information about the structure of the variety. More sensible would be to consider an infinite sequence of ever larger finite fields which probe the variety at ever smaller scales. It is this consideration which leads us to the concept of counting the number of solutions $N_{q^{r}}=\#\left(X / \mathbb{F}_{q^{r}}\right)$ and to ask what, if any, interesting information is provided by the numbers determined by the extensions $\mathbb{F}_{q^{r}}$ of degree $r$ of the finite field $\mathbb{F}_{q}$. The following paragraphs outline the strategy envisioned in this program and briefly introduce the key ingredients used in this paper. 
The starting point of the arithmetic considerations in Section 3 is to arrange the sequence of reductions of the variety over the finite fields $\mathbb{F}_{q^{r}}$ into a useful form. This can be achieved via Artin's congruent zeta function, essentially defining an exponential sum of a generating function constructed from the numbers $N_{q^{r}}$. For reasons just described we should not restrict the construction to local considerations at particular integers. Hence we need some way to pass to a global description in which all reference to fixed scales has been erased. This leads, in Section 4, to the concept of the global Hasse-Weil L-function of a Calabi-Yau variety. This L-function will collect the information at all rational primes. This step can be taken because the Weil conjectures show that in the present context Artin's zeta function is a rational function determined by the cohomology of the variety. The Hasse-Weil L-function therefore is a cohomological L-function.

At this point the only number theoretic algebraic structures that have appeared are the finite fields $\mathbb{F}_{q}$ and their extensions. A brief review of some of the aspects of the underlying conformal field theoretic aspects, provided in Section 2, reveals that this is not enough. As mentioned above, the structures that enter in Gepner's construction and its generalizations are rational, i.e. they lead to central charges and spectra of anomalous dimensions which are rational numbers. It is not clear how to recover these numbers from the intrinsic geometry of the variety. It turns out that it is more useful in the present context to encode the conformal field theoretic information in an alternative way by mapping the central charge and the anomalous dimensions via the Rogers dilogarithm into the quantum dimensions associated to the physical fields. These (generalized) quantum dimensions are elements of certain algebraic number fields which are determined by the fusion rules of the conformal field theory.

This suggests that we consider the structure of algebraic number fields in more detail. It is in this context that Hecke introduced a general notion of L-functions which are determined completely by the prime ideals of the number field, generalizing earlier results obtained by Dirichlet and Dedekind. Such number field L-functions essentially count the inverse of the norms of ideals in the number field weighted by a character of this field. 
The surprise now is that the cohomological Hasse-Weil type L-function of the Calabi-Yau variety in fact happens to be determined by a number field L-function of the type introduced by Hecke. This is the link that allows us to recover directly from the intrinsic geometry of the variety the number theoretic framework relevant to the underlying conformal field theory. Once the fusion field has been identified one can then further explore the arithmetic rôle played by the quantum dimensions in this field.

The strategy to be employed in this paper thus can be summarized as follows. First consider the arithmetic structure of Calabi-Yau varieties via Artin's congruent zeta function. Next define the global cohomological Hasse-Weil L-function via local factors from the congruent zeta function. Finally interpret the Hasse-Weil L-series as Hecke L-series of an algebraic number field. In this way we can recover the algebraic fusion field of the conformal field theory from the algebraic variety.

The program to use arithmetic geometry to illuminate the conformal field theoretic structure of Calabi-Yau varieties originated several years ago. The idea initially was to develop further some results derived by Bloch and Schoen in support of the Beilinson-Bloch conjectures and apply them in the context of the conformal field theory/Calabi-Yau relation [11]. More recently arithmetic considerations have been discussed in different contexts in refs. [12] and [13].

\section{Exactly solvable Calabi-Yau manifolds}

In the following we investigate the simplest class among weighted Calabi-Yau hypersurfaces described by higher-dimensional analogs of polynomials of Brieskorn-Pham type. These manifolds are distinguished by the fact that for every members there exists a particular point in moduli space for which the underlying exactly solvable conformal field theory is known explicitly. As indicated in (11) the varieties are described as zero-loci of polynomials in weighted projective space. 
The underlying rational conformal field theories suggested by Gepner are exactly solvable tensor products of $\mathrm{N}=2$ superconformal minimal models of central charge

$$
c=\frac{3 k}{k+2} \text {. }
$$

These factors are glued together by Gepner's construction [1], to which we refer for a more detailed discussion of the conformal field theory aspects. The physical string theoretic spectrum of the theory is determined by the conformal field theory chiral primary operators fields with integral anomalous dimension. These fields are constructed from operators in the individual $\mathrm{SU}(2)_{k}$ factors with the anomalous dimensions

$$
\Delta_{j}^{(k)}=\frac{j(j+2)}{4(k+2)}, \quad, j=0, \ldots, k .
$$

The SU(2) factors are the nontrivial interacting conformal field theories that provide the building blocks of the Gepner models.

It would thus appear that a possible, and perhaps natural, strategy should be an attempt to recover these rational numbers given by the underlying conformal field theory from the intrinsic geometry of the algebraic variety. It turns out however that it is more useful to proceed along a more indirect route in which the information of the anomalous dimensions and the central charge is first encoded in a set of numbers which are not elements of $\mathbb{Q}$, i.e. they are not rational. This will provide deeper information about the conformal field theory and is the route taken in this paper.

In order to establish this relation recall the modular $S$-matrix of the affine theories. Consider maps

$$
\chi: \mathfrak{H} \times \mathbb{C} \longrightarrow \mathbb{C}
$$

defined on the upper half plane $\mathfrak{H}$ and $\mathbb{C}$ parametrizing the charges of the extended theory

$$
\chi_{i}(\tau, u):=\operatorname{tr}_{\mathcal{H}_{i}} q^{L_{0}-\frac{c}{24}} e^{2 \pi i u J_{0}} .
$$

In the simple case of $\mathrm{SU}(2)_{k}$ the modular transformations

$$
\chi_{i}\left(-\frac{1}{\tau}, \frac{u}{\tau}\right)=e^{\pi i k u^{2} / 2} \sum_{j} S_{i j} \chi_{j}(\tau, u)
$$


lead to the modular $S$-matrix

$$
S_{i j}=\sqrt{\frac{2}{k+2}} \sin \left(\frac{(i+1)(j+1) \pi}{k+2}\right), \quad 0 \leq i, j \leq k .
$$

With these matrices one can define the generalized quantum dimensions as

$$
Q_{i j}=\frac{S_{i j}}{S_{0 j}}
$$

for the SU(2) theory at level $k$. The importance of these numbers derives from the fact that even though they do not directly provide the scaling behavior of the correlation functions, they do contain the complete information about the anomalous dimensions as well as the central charge

$$
\frac{1}{L(1)} \sum_{i=1}^{k} L\left(\frac{1}{Q_{i j}^{2}}\right)=\frac{3 k}{k+2}-24 \Delta_{j}^{(k)}+6 j,
$$

where $L$ is Rogers' dilogarithm

$$
L(z)=L i_{2}(z)+\frac{1}{2} \log (z) \log (1-z)
$$

and $L i_{2}$ is Euler's classical dilogarithm

$$
L i_{2}(z)=\sum_{n \in \mathbb{N}} \frac{z^{n}}{n^{2}}
$$

It follows that the quantum dimensions contain the essential information about the spectrum of the conformal field theory and Rogers' dilogarithm provides, via Euler's dilogarithm, the map from the quantum dimensions to the central charge and the anomalous dimensions. A review of these results and references to the original literature can be found in [14].

It is possible to gain insight into the nature of these particular numbers. The point here is that the extended quantum dimensions $Q_{i j}$ are elements of a particular field which extends the field of rational numbers. To determine the structure of this field one can proceed as follows. The starting point is the fusion algebra which describes a product of the chiral primary fields of the conformal field theory

$$
\Phi_{i} \star \Phi_{j}=\left(\mathcal{N}_{i}\right)_{j}^{k} \Phi_{k}
$$


where the resulting coefficients $\left(\mathcal{N}_{i}\right)_{j}^{k}$ define the so-called fusion matrices $\mathcal{N}_{i}$. The generalized quantum dimensions are eigenvalues of these fusion matrices and therefore they are solutions of the characteristic polynomials

$$
\operatorname{det}\left(Q \cdot \mathbf{1}-\mathcal{N}_{i}\right)=0
$$

Because the $\mathcal{N}_{i}$ are integral matrices the characteristic polynomial has integral coefficients and it is also normalized, i.e. its leading coefficient is unity. Therefore the $Q_{i j}$ are algebraic integers in some algebraic number field $K$ over the rational numbers $\mathbb{Q}$. An algebraic number field $K$ of degree $n=[K: \mathbb{Q}]$ is defined as the set of solutions of an equation

$$
a_{0}+a_{1} x+a_{2} x^{2}+\cdots+a_{n} x^{n}=0
$$

with coefficients $a_{i}$ which are rational integers $a_{i} \in \mathbb{Z}$. The set of algebraic integers of $K$ is defined as the set of solutions of equation (11) whose leading coefficient is unity $a_{n}=1$. The resulting expressions in general involve rational coefficients.

The extension $K / \mathbb{Q}$ is normal and because $\mathbb{Q}$ is of characteristic zero this extension is a Galois extension, i.e. its Galois group $\operatorname{Gal}(\mathrm{K} / \mathbb{Q})$ is abelian. Hence it follows from the theorem of Kronecker and Weber that the field $K$ is contained in some cyclotomic field $\mathbb{Q}\left(\mu_{m}\right)$ where $\mu_{m}$ is the cyclic group of order $m$, generated by the primitive $m$ 'th root of unity [15]. We will call the minimal cyclotomic field which contains all the quantum dimensions the fusion field of the rational conformal field theory.

In the present case we can be more specific: the generalized quantum dimensions take values $Q_{i j}\left(\mathrm{SU}(2)_{k}\right) \in \mathbb{Q}\left(\mu_{2(k+2)}\right)$ and because the quantum dimensions are real they are elements of a real subfield of the cyclotomic field. For the conformal field theory underlying the quintic hypersurface one finds e.g.

$$
Q_{i}\left(\mathrm{SU}(2)_{3}\right) \in\left\{1, \frac{1}{2}(1+\sqrt{5})\right\} \subset \mathbb{Q}(\sqrt{5})
$$

i.e. the fusion field of the affine conformal field theory underlying the quintic is the cyclotomic field $\mathbb{Q}\left(\mu_{5}\right)$. 
The problem of recovering the conformal field theory spectrum encoded by the anomalous dimensions can now be rephrased into the question whether it is possible to derive the fusion field of the quantum dimensions from the arithmetic structure of the corresponding CalabiYau manifold. Once this is achieved one can ask for an intrinsic field theoretic interpretation of the real quantum dimensions within the fusion field.

\section{Artin's Congruent Zeta Function of Calabi-Yau three- folds}

The starting point of the arithmetic analysis is the set of Weil conjectures [16], the proof of which was completed by Deligne [17]. For algebraic varieties the Weil-Deligne result states a number of structural properties for the congruent zeta function at a prime number $p$ defined as

$$
Z\left(X / \mathbb{F}_{p}, t\right) \equiv \exp \left(\sum_{r \in \mathbf{N}} \#\left(X / \mathbb{F}_{p^{r}}\right) \frac{t^{r}}{r}\right) .
$$

The motivation to arrange the numbers $N_{p, r}=\#\left(X / \mathbb{F}_{p^{r}}\right)$ in this unusual way originates from the fact that they often show a simple behavior, as a result of which the zeta function can be shown to be a rational function. This was first shown by Artin in the 1920s for hyperelliptic function fields. Further experience by Hasse, Weil, and others led to the conjecture that this phenomenon is more general, culminating in the Weil conjectures.

These general claims can be summarized as follows.

1. The zeta function of an algebraic variety of dimension $d$ satisfies a functional equation 18

$$
Z\left(X / \mathbb{F}_{p}, \frac{1}{p^{d} t}\right)=(-1)^{\chi+\mu} p^{d \chi / 2} t^{\chi} Z\left(X / \mathbb{F}_{p}, t\right),
$$

where $\chi$ is the Euler number of the variety $X$ over the complex numbers $\mathbb{C}$. Furthermore $\mu$ is zero when the dimension $d$ of the variety is odd, and $\mu$ is the multiplicity of $-p^{d / 2}$ as 
an eigenvalue of the action induced on the cohomology by the Frobenius automorphism $\Phi: X \longrightarrow X, x \mapsto x^{p}$.

2. $Z\left(X / \mathbb{F}_{p}, t\right)$ is a rational function which can be written as

$$
Z\left(X / \mathbb{F}_{p}, t\right)=\frac{\prod_{j=1}^{d} \mathcal{P}_{2 j-1}^{(p)}(t)}{\prod_{j=0}^{d} \mathcal{P}_{2 j}^{(p)}(t)}
$$

where

$$
\mathcal{P}_{0}^{(p)}(t)=1-t, \quad \mathcal{P}_{2 d}^{(p)}(t)=1-p^{d} t
$$

and for $1 \leq i \leq 2 d-1$

$$
\mathcal{P}_{i}^{(p)}(t)=\prod_{j=1}^{b_{i}}\left(1-\beta_{j}^{(i)}(p) t\right),
$$

with algebraic integers $\beta_{j}^{(i)}(p)$. The $b_{i}$ denote the Betti numbers of the variety, $b_{i}=$ $\operatorname{dim} \mathrm{H}_{\text {DeRham }}^{i}(X)$. The rationality of the zeta function was first shown by Dwork [19 by adelic methods.

3. The algebraic integers $\beta_{j}^{(i)}(p)$ satisfy the Riemann hypothesis

$$
\left|\beta_{j}^{(i)}(p)\right|=p^{i / 2}, \quad \forall i
$$

It is this part of the Weil conjectures which resisted the longest, and was finally proved by Deligne.

We are interested in Calabi-Yau threefolds with finite fundamental group, i.e. Calabi-Yau varieties with $h^{1,0}=0=h^{2,0}$. For such varieties the expressions above simplify considerably. For an arbitrary Calabi-Yau threefold the congruent zeta function takes the form

$$
Z\left(X / \mathbb{F}_{p}, t\right)=\frac{\mathcal{P}_{3}^{(p)}(t)}{(1-t) \mathcal{P}_{2}^{(p)}(t) \mathcal{P}_{4}^{(p)}(t)\left(1-p^{3} t\right)}
$$

with

$$
\operatorname{deg}\left(\mathcal{P}_{3}^{(p)}(t)\right)=2+2 h^{(2,1)}, \quad \operatorname{deg}\left(\mathcal{P}_{2}^{(p)}(t)\right)=h^{(1,1)}
$$


This follows from the fact that for non-toroidal Calabi-Yau threefolds we have $b_{1}=0$. In general the indicated polynomials $\mathcal{P}_{i}^{(q)}(t)$ left unspecified are non-trivial for the BrieskornPham varieties of interest in the present context. This nontrivial structure arises from the fact that weighted projective varieties admit singularities which have to be resolved and therefore lead to contributions of $H^{2}(X)$.

The relation between the Calabi-Yau varieties and conformal field theories is universal for all members of the Brieskorn-Pham type, independent on whether they had to be resolved or were smooth initially. This suggests that it must be possible to recover the basic ingredients necessary to derive the conformal field theory already from the class of smooth manifolds. For varieties which do not have to be resolved the structure of the zeta functions is particularly simple. In particular one finds that the congruent zeta function of smooth Calabi-Yau hypersurfaces in weighted projective fourspace is given by

$$
Z\left(X / \mathbb{F}_{p}, t\right)=\frac{\mathcal{P}_{3}^{(p)}(t)}{(1-t)(1-p t)\left(1-p^{2} t\right)\left(1-p^{3} t\right)}
$$

with

$$
\operatorname{deg}\left(\mathcal{P}_{3}^{(p)}(t)\right)=2+2 h^{(2,1)}
$$

and

$$
\sum_{i=1}^{b_{3}} \beta_{i}^{(3)}=1+p+p^{2}+p^{3}-\#\left(X / \mathbb{F}_{p}\right) .
$$

Hence for smooth hypersurface threefolds the only interesting information of the zeta function is encoded in the polynomials $\mathcal{P}_{3}^{(p)}(t)$.

\section{Hasse-Weil L-function}

We see from the rationality of the zeta function that the basic information of this quantity is parametrized by the cohomology of the variety. More precisely, one can show that the $i$ 'th polynomial $\mathcal{P}_{i}^{(p)}(t)$ is associated to the action induced by the Frobenius morphism on the $i$ 'th

cohomology group $\mathrm{H}^{i}(X)$. In order to gain insight into the arithmetic information encoded in 
this Frobenius action it is useful to decompose the zeta function. This leads to the concept of a local L-function that is associated to the polynomials $\mathcal{P}_{i}^{(p)}(t)$ via the following definition.

Let $\mathcal{P}_{i}^{(p)}(t)$ be the polynomials determined by the rational congruent zeta function over the field $\mathbb{F}_{p}$. The $i$ 'th L-function of the variety $X$ over $\mathbb{F}_{p}$ then is defined via

$$
L^{(i)}\left(X / \mathbb{F}_{p}, s\right)=\frac{1}{\mathcal{P}_{i}^{(p)}\left(p^{-s}\right)}
$$

Such L-functions are of interest for a number reasons. One of these is that often they can be modified by simple factors so that after analytic continuation they (are conjectured to) satisfy some type of functional equation.

It was mentioned in the previous section that the geometry/CFT relation must hold for the simplest type of varieties, in particular those that do not need any kind of resolution. When considering the cohomology of such simple varieties in dimensions one through four it becomes clear that independent of the dimension of the Calabi-Yau variety the essential ingredient is provided by the cycles that span the middle-dimensional (co)homology. Hence even though in general the cohomology can be fairly complex, in particular for Calabi-Yau fourfolds, the only local factor in the L-function that is relevant for the present discussion is $\mathcal{P}_{d}^{(p)}(t)$ for $d=\operatorname{dim}_{\mathbb{C}} X$

This suggests a natural generalization of the concept of the Hasse-Weil L-function of an elliptic curve. Let $X$ be a Calabi-Yau $d$-fold with $h^{i, 0}=0$ for $0<i<n-1$ and denote by $P(X)$ the set of good prime numbers of $X$, i.e. those prime numbers for which the variety has good reduction over $\mathbb{F}_{p}$. Then its associated Hasse-Weil L-function is defined as

$$
L_{\mathrm{HW}}(X, s)=\prod_{p \in P(X)} \frac{1}{\mathcal{P}_{d}^{(p)}\left(p^{-s}\right)}=\prod_{p \in P(X)} \frac{1}{\prod_{j=1}^{b_{3}}\left(1-\beta_{j}^{(d)}(p) p^{-s}\right)} .
$$

The restriction to good primes is a nontrivial requirement. Even though the Brieskorn-Pham type varieties are all smooth (i.e. transverse) as varieties over the complex numbers $\mathbb{C}$ they do not retain this property for arbitrary subfields $K \subset \mathbb{C}$. In the case of the quintic considered 
below the prime $p=5$ is an example of a bad prime. The existence of bad primes complicates the whole theory considerably, but for our purposes we can ignore the additional factors of the completed L-functions that are induced by these bad primes.

As mentioned above, for smooth weighted CY hypersurfaces the Hasse-Weil L-function then contains the complete arithmetic information of the congruent zeta function. For reasons that will become clear below it is of importance that the above Hasse-Weil function can be related to a Hecke L-function, induced by Hecke characters. The main virtue of such characters is that as the simpler Dirichlet characters they are multiplicative maps. It is this multiplicativity which is of essence for the present framework.

\subsection{The Quintic Example}

Consider the Calabi-Yau variety defined by the quintic hypersurface in ordinary projective fourspace $\mathbb{P}_{4}$. We denote the general $h^{2,1}=101$ complex dimensional family of quintic hypersurfaces in projective fourspace $\mathbb{P}_{4}$ by $\mathbb{P}_{4}[5]$ and consider the threefold defined by

$$
\mathbb{P}_{4}[5] \ni X=\left\{\sum_{i=0}^{4} x_{i}^{5}=0\right\} .
$$

It follows from Lefshetz's hyperplane theorem that the cohomology below the middle dimension is inherited from the ambient space. Thus we have $h^{1,0}=0=h^{0,1}$ and $h^{1,1}=1$ while $h^{2,1}=101$ follows from counting monomials of degree five. Following Weil [16] the zeta function is determined by (23), where the numerator is given by the polynomial $\mathcal{P}_{3}^{(p)}(t)=$ $\prod_{i=1}^{204}\left(1-\beta_{i}^{(3)}(p) t\right)$ which takes the form

$$
\mathcal{P}_{3}^{(p)}(t)=\prod_{\alpha \in \mathcal{A}}\left(1-j_{p}(\alpha) t\right) .
$$

This expression involves the following ingredients. Define $\ell=(5, p-1)$ and rational numbers $\alpha_{i}$ via $\ell \alpha_{i} \equiv 0(\bmod 1)$. The set $\mathcal{A}$ is defined as

$$
\mathcal{A}=\left\{\alpha=\left(\alpha_{0}, \ldots, \alpha_{4}\right) \mid 0<\alpha_{i}<1,5 \alpha_{i} \equiv 0(\bmod 1), \sum_{i} \alpha_{i}=0(\bmod 1)\right\} .
$$


Defining the characters $\chi_{\alpha_{i}} \in \hat{\mathbb{F}}_{p}$ in the dual of $\mathbb{F}_{p}$ as $\chi_{\alpha_{i}}\left(u_{i}\right)=\exp \left(2 \pi i \alpha_{i} s_{i}\right)$ with $u_{i}=g^{s_{i}}$ for a generating element $g \in \mathbb{F}_{p}$, the factor $j_{p}(\alpha)$ finally is determined as

$$
j_{p}(\alpha)=\frac{1}{p-1} \sum_{\sum_{i} u_{i}=0} \prod_{i=0}^{4} \chi_{\alpha_{i}}\left(u_{i}\right) .
$$

We thus see that the congruent zeta function leads to the Hasse-Weil L-function associated to a Calabi-Yau threefold

$$
L_{\mathrm{HW}}(X, s)=\prod_{p \in P(X)} \prod_{\alpha \in \mathcal{A}}\left(1-\frac{j_{p}(\alpha)}{p^{s}}\right)^{-1},
$$

ignoring the bad primes, which are irrelevant for our purposes.

\section{L-Functions and Algebraic Number Fields}

As mentioned in the Introduction, the surprising aspect of the Hasse-Weil L-function is that it is determined by another, a priori completely different kind of L-function that is derived not from a variety but from a number field. It is this possibility to interpret the cohomological Hasse-Weil L-function as a field theoretic L-function which establishes the connection that allows to derive number fields $K$ from algebraic varieties $X$.

For the case at hand the type of L-function that is relevant is that of a Hecke L-function determined by a Hecke character, more precisely an algebraic Hecke character. Following Weil we will see that the relevant field for our case is the extension $\mathbb{Q}\left(\mu_{m}\right)$ of the rational integers $\mathbb{Q}$ by roots of unity, generated by $\xi=e^{2 \pi i / m}$ for some rational integer $m$. It turns out that these fields fit in very nicely with the conformal field theory point of view. In order to see how this works this Section first describes the concept of Hecke characters and then explains how the L-function fits into this framework.

There are many different different definitions of algebraic Hecke characters, depending on the precise number theoretic framework. Originally this concept was introduced by Hecke 
[20] as Grössencharaktere of an arbitrary algebraic number field. In the following Deligne's adaptation of Weil's Grössencharaktere of type $A_{0}$ is used [21]. Let $\mathcal{O}_{K} \subset K$ be the ring of integers of the number field $K, \mathfrak{f} \subset \mathcal{O}_{K}$ an integral ideal, and $E$ a field of characteristic zero. Denote by $\mathfrak{I}_{\mathfrak{f}}(K)$ the set of fractional ideals of $K$ that are prime to $\mathfrak{f}$ and denote by $\mathfrak{P}_{\mathfrak{f}}(K)$ the principal ideals $(\alpha)$ of $K$ for which $\alpha \equiv 1(\bmod \mathfrak{f})$. An algebraic Hecke character modulo $\mathfrak{f}$ is a multiplicative function $\chi$ defined on the ideals $\mathfrak{I}_{\mathfrak{f}}(K)$ for which the following condition holds. There exists an element in the integral group ring $\sum n_{\sigma} \sigma \in \mathbb{Z}[\operatorname{Hom}(K, \bar{E})]$, where $\bar{E}$ is the algebraic closure of $E$, such that if $(\alpha) \in \mathfrak{P}_{\mathfrak{f}}(K)$ then

$$
\chi((\alpha))=\prod_{\sigma} \sigma(\alpha)^{n_{\sigma}} .
$$

Furthermore there is an integer $w>0$ such that $n_{\sigma}+n_{\bar{\sigma}}=w$ for all $\sigma \in \operatorname{Hom}(K, \bar{E})$. This integer $w$ is called the weight of the character $\chi$.

Given any such character $\chi$ defined on the ideals of the algebraic number field $K$ we can follow Hecke and consider a generalization of the Dirichlet series via the L-function

$$
L(\chi, s)=\prod_{\substack{\mathfrak{p} \subset \mathcal{O}_{K} \\ \mathfrak{p} \text { prime }}} \frac{1}{1-\frac{\chi(\mathfrak{p})}{\mathrm{Np}^{s}}}=\sum_{\mathfrak{a} \subset \mathcal{O}_{K}} \frac{\chi(\mathfrak{a})}{\mathrm{Na}^{s}}
$$

where the sum runs through all the ideals. Here $\mathrm{Np}$ denotes the norm of the ideal $\mathfrak{p}$, which is defined as the number of elements in $\mathcal{O}_{K} / \mathfrak{p}$. The norm is a multiplicative function, hence can be extended to all ideals via the prime ideal decomposition of a general ideal. If we can deduce from the Hasse-Weil L-function the particular Hecke character(s) involved we will be able to derive directly from the variety in an intrinsic way distinguished number field(s) $K$.

Insight into the nature of number fields can be gained by recognizing that for certain extensions $K$ of the rational number $\mathbb{Q}$ the higher Legendre symbols provide the characters that enter the discussion above. Inspection then suggests that we consider the power residue symbols of cyclotomic fields $K=\mathbb{Q}\left(\mu_{m}\right)$ with integer ring $\mathcal{O}_{K}=\mathbb{Z}\left[\mu_{m}\right]$. The transition from the cyclotomic field to the finite fields is provided by the character which is determined for any 
algebraic integer $x \in \mathbb{Z}\left[\mu_{m}\right]$ prime to $m$ by the map

$$
\chi_{\bullet}(x): \mathfrak{I}_{m}\left(\mathcal{O}_{K}\right) \longrightarrow \mathbb{C}^{*},
$$

which is defined on ideals $\mathfrak{p}$ prime to $m$ by sending the prime ideal to the $m$ 'th root of unity for which

$$
\mathfrak{p} \mapsto \quad \chi_{\mathfrak{p}}(x)=x^{\frac{\mathrm{Np}-1}{m}}(\bmod \mathfrak{p})
$$

Using these characters one can define Jacobi-sums of rank $r$ for any fixed element $a=$ $\left(a_{1}, \ldots, a_{r}\right)$ by setting

$$
J_{a}^{(r)}(\mathfrak{p})=(-1)^{r+1} \sum_{\substack{u_{i} \in \mathcal{O}_{K} / \mathfrak{p} \\ \sum_{i} u_{i}=-1(\bmod \mathfrak{p})}} \chi_{\mathfrak{p}}\left(u_{1}\right)^{a_{1}} \cdots \chi_{\mathfrak{p}}\left(u_{r}\right)^{a_{r}}
$$

for prime $\mathfrak{p}$. For non-prime ideals $\mathfrak{a} \subset \mathcal{O}_{K}$ the sum is generalized via prime decomposition $\mathfrak{a}=\prod_{i} \mathfrak{p}_{i}$ and multiplicativity $J_{a}(\mathfrak{a})=\prod_{i} J_{a}\left(\mathfrak{p}_{i}\right)$. Hence we can interpret these Jacobi sums as a map $J^{(r)}$ of rank $r$

$$
J^{(r)}: \mathfrak{I}_{m}\left(\mathbb{Z}\left[\mu_{m}\right]\right) \times(\mathbb{Z} / m \mathbb{Z})^{r} \longrightarrow \mathbb{C}^{*}
$$

where $\mathfrak{I}_{m}$ denotes the ideals prime to $m$. For fixed $\mathfrak{p}$ such Jacobi sums define characters on the group $(\mathbb{Z} / m \mathbb{Z})^{r}$. It can be shown that for fixed $a \in(\mathbb{Z} / m \mathbb{Z})^{r}$ the Jacobi sum $J_{a}^{(r)}$ evaluated at principal ideals $(x)$ for $x \equiv 1\left(\bmod m^{r}\right)$ is of the form $x^{S(a)}$, where

$$
S(a)=\sum_{\substack{(\ell, m)=1 \\ \bmod m}}\left[\sum_{i=1}^{r}\left\langle\frac{\ell a_{i}}{m}\right\rangle\right] \sigma_{-\ell}^{-1}
$$

where $\langle x\rangle$ denotes the fractional part of $x$ and $[x]$ describes the integer part of $x$.

We therefore see that the Hasse-Weil L-function is in fact a product of functions each of which is determined by a Hecke character defined by a Jacobi sum that is determined by a prime ideal in the cyclotomic field $\mathbb{Q}\left(\mu_{m}\right)$. In the case of the quintic hypersurface we derive in this way the fusion field from the arithmetic structure of the defining variety reduced to a finite field. To summarize, we have seen that the fusion field of the underlying conformal field theory is precisely that number field which is determined when the cohomological Hasse-Weil L-function is interpreted as the Hecke L-function associated to an algebraic number field. 


\section{Quantum dimensions and class number}

At this point we have identified the fusion field with the number field associated to the Hecke L-function, which in turn is associated to the Hasse-Weil L-function. In order to extend the dictionary between conformal field theoretic quantities and number theoretic quantities we can ask whether we can also obtain a number theoretic interpretation for the quantum numbers which take values in the fusion field. This amounts to the question whether these quantum numbers form some particular substructure of the cyclotomic field which can be singled out for purely number theoretic reason.

In order to see that this is indeed possible consider first the so-called class number $h$ of a cyclotomic field $\mathbb{Q}\left(\mu_{m}\right)$. This number plays an essential role in illuminating the structure of general algebraic number fields, but our focus will be on the cyclotomic fields [22] [23]. The class number of an algebraic number field $K$ can be defined as the number of ideal classes formed by the following equivalence relation. Two ideals are considered equivalent if they can be made identical by the multiplication of principal ideals, the latter being generated by single

algebraic numbers. The set of such ideal classes forms a finite group whose order is defined to be the class number. Put slightly differently we can think about the ideal class group $\mathrm{Cl}\left(\mathcal{O}_{K}\right)$ as the quotient of the group of fractional ideals

$$
\mathcal{F}=\left\{\frac{\mathfrak{a}_{1}}{\mathfrak{a}_{2}} \mid \mathfrak{a}_{1}, \mathfrak{a}_{2} \subset \mathcal{O}_{K}, \mathfrak{a}_{1}, \mathfrak{a}_{2} \neq 0\right\}
$$

of the ring $\mathcal{O}_{K}$ of algebraic integers in $K$ by the group of non-zero principal ideals

$$
\mathcal{P}=\left\{(a)=a \mathcal{O}_{K} \mid a \in K^{\times}\right\}
$$

The importance of the class number $h(K)=\# \mathrm{Cl}\left(\mathcal{O}_{K}\right)$ derives from the fact that it measures how close a number field comes to unique prime factorization. As an example consider the rational numbers $\mathbb{Q}$. Its ring of integers $\mathbb{Z}$ is a principal ring, hence its class number is unity. Furthermore we have unique prime factorization in $\mathbb{Z}$. It turns out that analogous relations hold in general for algebraic number fields, i.e. the class number $h(K)$ is unity if and only if there is unique factorization. 
Of essence for the very concept of unique prime factorization are the so-called units of the field. Such units generalize the units in the ring of rational integers $\{ \pm 1\} \subset \mathbb{Z}$, and are algebraic integers whose inverses are again algebraic integers. Unique factorization is always meant modulo units. The set of units in an algebraic field $K$ defines a subgroup $U \subset K$.

Coming back to the cyclotomic fields of interest in the present context, one finds that the class number of these fields exhibit a composite structure. It is known that this class number is always a composite number

$$
h\left(\mathbb{Q}\left(\mu_{p}\right)\right)=h^{+} h^{*},
$$

where $h^{+}$itself is the class number of the real cyclotomic subfield of $\mathbb{Q}\left(\mu_{p}\right)$.

Putting together the concepts introduced in the above paragraphs provides a number theoretic identification of the quantum dimensions. Namely, it turns out that the class number $h^{+}$of the real subfield of $\mathbb{Q}\left(\mu_{m}\right)$ admits an interpretation as the index of a subgroup within the maximal real subfield in the cyclotomic field. Let $2<p \neq 2(\bmod 4)$ be the conductor of $\mathbb{Q}\left(\mu_{p}\right)$ and denote by $U^{+}$the group of positive real units in $\mathbb{Q}\left(\mu_{p}\right)$, i.e. the positive real invertible elements in the cyclotomic field. Let further denote $U_{c}^{+}$the subgroup spanned by the cyclotomic units $\theta_{r}$ within $U^{+}$

$$
\theta_{r}=\left|\frac{1-\xi^{r}}{1-\xi}\right|=\frac{\sin \frac{r \pi}{p}}{\sin \frac{\pi}{p}}
$$

Then

$$
h^{+}=2^{-b}\left[U^{+}: U_{c}^{+}\right],
$$

where $b=0$ if the number $f$ of prime factors is unity, and $b=2^{f-2}+1-f$ if $f>1$ [24]. This provides an identification of the quantum dimensions within the fusion field.

One can further show that the class number $h^{+}$of the real subfield of $\mathbb{Q}\left(\mu_{p}\right)$ is in fact partially constructed by the quantum dimensions. Let $p$ be an odd prime and denote by $\sigma_{j}$ the elements $\xi_{p} \mapsto \xi_{p}^{g^{j}}$ of the Galois group $\operatorname{Gal}\left(\mathbb{Q}\left(\mu_{p}\right) / \mathbb{Q}\right)$ of $\mathbb{Q}\left(\mu_{p}\right)$ generated by a primitive root modulo $p$. Then

$$
h^{+}=\frac{2^{\frac{p-3}{2}} \Delta}{R}
$$


where the determinant $\Delta$ is constructed from the quantum dimensions as

$$
\Delta=\left|\operatorname{det}\left(\sigma_{j}\left(\theta_{k}\right)\right)_{\substack{2 \leq k \leq(p-1) / 2 \\ 0 \leq j \leq(p-3) / 2}}\right| .
$$

The regulator $R$ can be viewed as the volume of the logarithmic image of a fundamental system of units. It was shown by Dirichlet that the group $U$ of units in an algebraic number field of degree $[K: \mathbb{Q}]=r_{1}+2 r_{2}$ takes the form

$$
U \cong \mu \prod_{i=1}^{r_{1}+r_{2}-1} G_{i}
$$

where $\mu$ is the group of roots of unity, each $G_{i}$ is a group of infinite order, and $r_{1}\left(r_{2}\right)$ denotes the number of real (complex) embeddings of the field $K$. Hence every unit $u \in U$ can be written in the form $u=\alpha \prod_{r=1}^{r} \epsilon_{i}$, where $\alpha \in \mu$ and $\left\{\epsilon_{i}\right\}_{i=1, \ldots, r=r_{1}+r_{2}-1}$ is called a fundamental system of units. It is useful to translate the multiplicative structure of the units into an additive framework via the regulator map

$$
r: U \longrightarrow \mathbb{R}^{r_{1}+r_{2}}
$$

defined by

$$
r(u)=\left(\ln \left|\rho_{1}(u)\right|, \ldots, \ln \left|\rho_{r_{1}}(u)\right|, \ln \left|\rho_{r_{1}+1}(u)\right|^{2}, \ldots, \ln \left|\rho_{r_{1}+r_{2}}(u)\right|^{2}\right),
$$

where $\left\{\rho_{i}\right\}_{i=1, \ldots, r_{1}}$ are the real embeddings and $\left\{\rho_{r_{1}+j}\right\}_{j=1, \ldots, r_{2}}$ are the complex embeddings of $K$. The regulator $R$ then is defined as

$$
R=\operatorname{det}\left(a_{i} \ln \left|\rho_{i}\left(\epsilon_{j}\right)\right|\right) \underset{\substack{1 \leq i \leq r_{1}+r_{2} \\ 1 \leq j \leq r_{1}+r_{2}-1}}{\substack{1 \\ 1}}
$$

with $a_{i}=1$ for the real embeddings $i=1, \ldots, r_{1}$ and $a_{i}=2$ for the complex embeddings $i=r_{1}+1, \ldots, r_{1}+r_{2}$. The regulator is independent of the choice of the fundamental system of units.

The results described above provide an example where the class number of an algebraic number field acquires physical significance. This is not without precedence. Recently the class 
number of the fields of definition of certain arithmetic black hole attractor varieties have been interpreted as the number of U-duality classes of black holes with the same area [12].

To summarize, we see that a further entry in our dictionary is provided by the identification of the quantum dimensions of the fusion field with the real cyclotomic units of the field that is determined by recognizing the Hasse-Weil L-function as the Hecke L-function of an algebraic number field.

\section{Acknowledgement}

This work has been done over a number years at several institutions. I'm grateful to Werner Nahm and his group at the University of Bonn for discussions during its first period. It is a pleasure to thank Alan Adolphson, Monika Lynker, Chad Schoen, John Stroyls, John Tate, Alexandre Varchenko, Katrin Wendland, and in particular Vipul Periwal, for discussions. Parts of this work were completed at UT Austin and during the Duality Workshop at the Institute for Theoretical Physics in Santa Barbara. I'm grateful to the Theory Group at Austin and the ITP for hospitality. This research was supported in part by NATO under grant CRG 9710045, an ITP scholarship, and the National Science Foundation under grant No. PHY99-07949.

\section{References}

[1] D.Gepner, Spacetime Supersymmetry in Compactified String Theory and Superconformal Models, Nucl.Phys. B296(1988)757;

String Theory and Calabi-Yau Manifolds: The Three Generation Case, preprint Dec. 1987, hep-th/9301089

[2] M.Lynker and R.Schimmrigk, ADE Quantum Calabi-Yau Manifolds, Nucl.Phys. B339 (1990) 121 
[3] J.Fuchs, A.Klemm, C.Scheich and M.G.Schmidt, Spectra and Symmetries of Gepner Models Compared to Calabi-Yau Manifolds, Ann.Phys. 204(1990)1

[4] P.Candelas, G.Horowitz, A.Strominger and E.Witten, Vaccum Configurations for Superstrings, Nucl.Phys. B258(1985)46

[5] R.Schimmrigk, A New Construction of a Three Generation Calabi-Yau Manifold, Phys.Lett. B193 (1987) 175

[6] E.Martinec, Algebraic Geometry and Effective Lagrangians, Phys.Lett. B217(1989)431

[7] C.Vafa and N.Warner, Catastrophes and the Classification of Conformal Field Theories, Phys.Lett. B218(1989)51

[8] W.Lerche, C.Vafa and N.Warner, Chiral Rings in N=2 Superconformal Theories, Nucl. Phys. B324 (1989) 427

[9] C. Vafa, String Vacua and Orbifoldized L-G Vacua, Mod.Phys.Lett. A4(1989)1169

[10] E.Witten, Phases of N=2 Theories in Two in Two Dimensions, Nucl. Phys. B403 (1993) 159, hep-th/9301042

[11] R. Schimmrigk, Lecture at Bonn University, 1995;

Lecture at the Workshop on Arithmetic, Geometry and Physics around Calabi-Yau Manifolds and Mirror Symmetry, J.D. Lewis and N. Yui, 2001

[12] G. Moore, Attractors and Arithmetic, hep-th/9807056; Arithmetic and Attractors, hepth/9807087

[13] P. Candelas, X. de la Ossa and F. Rodriguez-Villegas, Calabi-Yau Manifolds over Finite Fields I, hep-th/0012233;

P. Candelas, X. de la Ossa and F. Rodriques-Villegas, in preparation

[14] A.Kirillov, Dilogarithm Identities, Progr. Theor. Phys. Suppl 118 (1995) 61, hepth/9408113 
[15] J. de Boer and J. Goeree, Markov Traces and II(1) Factors in Conformal Field Theories, Commun. Math. Phys. 139 (1991) 267

[16] A.Weil, Number of solutions of equations in finite fields, Bull.Am.Math.Soc. 55(1949)497; Gött.Nachr. 1(1974)14

[17] P.Deligne, La conjecture de Weil I, Publ.Math. IHES 43(1974)273

[18] S.L.Kleiman, The standard conjectures, Proc. Symp. Pure Math. 55 (1994) 3

[19] B.Dwork, On the rationality of the zeta function of an algebraic variety, Amer.J.Math. $\mathbf{8 2}(1960) 631$

[20] E. Hecke, Eine neue Art von Zetafunktionen und ihre Beziehungen zur Verteilung der Primzahlen, Math. Z. 1(1918)357;

Eine neue Art von Zetafunktionen und ihre Beziehungen zur Verteilung der Primzahlen. Zweite Mitteilung, Math. Z. 6 (1920)11

[21] P.Deligne, Cohomologie étale, Springer Verlag LNM 569, 1977

[22] I.Stewart and D.Tall, Algebraic Number Theory, Chapman and Hall, 1979

[23] P. Ribenboim, Classical Theory of Algebraic Numbers, Springer Verlag, 2001

[24] W. Sinnott, On the Stickelberger Ideal and the Circular Units of a Cyclotomic Field, Ann. Math. 108 (1978) 107 